\title{
Effect of Seed Priming on the Activity of Antioxidant Enzymes and Changes of Biochemicals of Rice under Timely and Late Sown Conditions
}

\author{
Mahesh Kumar $^{1 *}$, Ravi P. Singh ${ }^{2}$ and Bandana Bose ${ }^{1}$ \\ ${ }^{1}$ Department of Plant Physiology, ${ }^{2}$ Department of Genetics and Plant Breeding, Institute of \\ Agricultural Sciences, Banaras Hindu University, Varanasi-221005, India \\ *Corresponding author
}

\section{A B S T R A C T}

\begin{tabular}{|l|}
\hline Ke y w o r d s \\
Seed Priming \\
Antioxidant Enzymes \\
Biochemicals \\
Rice
\end{tabular}

\section{Introduction}

Rice is a most important food crop, nearly one-half of the world population, including all East and Southeast-Asia completely dependent upon rice. The world population is increasing continuously day by day while Agricultural land is decreasing due to rapid urbanisation. Providing food to growing population is became a challenge in future for agricultural scientist. The sowing time is one of the most important yield deciding factor of rice. Early sowing of rice crop got more time for vegetative growth and development that lead to high yield and delay in sowing date causes significant reduction in growth and yield
(Abou Khalifa 1996; Sharief et al., 2000; Abou Khalifa 2005).Seed holds a key position in plant science because it is the main source of food supply. Further a hand full of seed is always easy to handle rather than 100 plants growing in the field. Hence seed research always attracts plant scientists. During germination the seed of any crop suffers from many kind of stresses may be biotic or abiotic because the soil itself is a harbour of many kind of stresses. Germination status decides the fate of the growing crop and finally the yield. Seed priming is a technique that improves seed germination, germination speed, seedling vigor, root length, seedling dry weight, photosynthetic efficiency and many 
other plant growth traits. Other than this it also improve biochemical status of plant by improving $\alpha$ - amylase activity and soluble sugar contents during seed germination, SOD, nitrate reductase activity (Anaytullah and Bose 2007; Sananda and Bose, 2012; Kumar et al., 2016). Seed priming enhanced seed germination and seedling vigor under adverse conditions in diverse species, such as soybean, maize, spinach, mustard, pepper and wheat and rice etc. (Bose et al.2007, Iqbal and Ashraf 2007, Farooq et al., 2008, Krishnottar et al., 2009, Korkmaz and Korkmaz, 2009, Zhuo et al., 2009, Chen et al., 2011, Srivastava and Bose2012). Pre-soaking and priming improved seedling establishment in flooded soil, enhanced the capacity to scavenge reactive oxygen species in seeds by increasing CAT and SOD activities, and enhanced carbohydrate mobilization. Seed priming treatment exhibit increased germination rate, greater germination uniformity, and greater total germination percentage (Sananda and Bose, 2012).

\section{Materials and Methods}

Rice variety HUR 105 was selected for experimental purpose. Healthy and bold seeds were surface sterilized by keeping them in $0.01 \% \mathrm{HgCl}_{2}$ (Mercuric chloride) solution for 5 minutes and then washed with distilled water for 4-5 times. Three types of priming treatments were used for this experiment included hydropriming $\left(\mathrm{T}_{1}\right)$ (using distilled water), halopriming and hormonal priming using $5 \mathrm{mM} / \mathrm{ppm}$ concentration of $\mathrm{Mg}\left(\mathrm{NO}_{3}\right)_{2}$ $\left(\mathrm{T}_{2}\right)$, Kinetin $\left(\mathrm{T}_{3}\right)$ and $0.75 \mathrm{mMconcentration}$ of salicylic $\operatorname{acid}\left(\mathrm{T}_{4}\right)$ respectively for $18 \mathrm{~h}$. A set containing non primed control seeds $\left(\mathrm{T}_{0}\right)$ was used during experimentation. After $18 \mathrm{~h}$ these seeds were air dried under fan to bring back to its initial weight further the seeds were kept in paper bags and used within one month. All parameters were taken at 20, 40, 60 and 80 DAT (Days after transplanting). Experiments were conducted in Factorial Randomized Block Design (RBD) during 2015 and 2016 at Agricultural Research Farm, Banaras Hindu University, Varanasi.

\section{Estimation of superoxide dismutase (SOD) activity $\left(\mathrm{U} \mathrm{g} \mathrm{g}^{-1} \mathrm{FWmin}^{-1}\right)$}

Superoxide dismutase activity was assayed by the method of Dhindsa et al., (1981).

\section{Preparation of enzyme extract}

Enzyme extract for SOD was prepared by first freezing the weighed amount of leaf samples $(1.0 \mathrm{~g})$ in ice basket to prevent proteolytic activity. The sample was grounded with $10 \mathrm{~mL}$ of extraction buffer $(0.1 \mathrm{M}$ phosphate buffer, $\mathrm{pH} \quad 7.5$ containing $(0.5 \mathrm{mM}$ EDTA). The grinded sample was centrifuged in cooling centrifuge machine at $10000 \mathrm{~g}$ for 10 minutes, after centrifugation supernatant was collected and this supernatant was used as enzyme source.

\section{Assay of SOD activity}

$3 \mathrm{~mL}$ of the reaction mixture containing $0.1 \mathrm{~mL}$ of $1.5 \mathrm{M}$ sodium carbonate, $0.2 \mathrm{~mL}$ of $200 \mathrm{mM}$ methionine, $0.1 \mathrm{~mL}$ of $2.25 \mathrm{mM}$ NBT, $0.1 \mathrm{~mL}$ of $3 \mathrm{mM}$ EDTA, $1.5 \mathrm{~mL}$ of $100 \mathrm{mM}$ potassium phosphate buffer, $1 \mathrm{~mL}$ of distilled water and $0.1 \mathrm{~mL}$ of enzyme extract were taken in test tubes in duplicate from each enzyme sample. Two tubes without enzyme extract were taken as control. The reaction was started by adding $0.1 \mathrm{~mL}$ riboflavin $(60 \mu \mathrm{M})$ and placing the tubes below a light source of two $15 \mathrm{~W}$ florescent lamps for 15 minutes. Reaction was stopped by switching off the light and covering the tubes by black cloth. Tubes without enzyme extract developed maximum colour. A non-irradiated complete mixture that did not develop colour served as blank. Absorbance was recorded at $560 \mathrm{~nm}$ in spectrophotometer. 
Enzyme Unit $(\mathrm{EU})=$

Where,

$(-)=$ Without enzyme

$(+)=$ With enzyme

The EU was expressed on per $\mathrm{g}$ fresh weight basis.

\section{Determination of nitrate reductase activity (NRA)}

In vivo activities of enzyme nitrate reductase of green leaves of rice were determined by the method of Srivastava (1974).

\section{Estimation of proline Content ( $\left.\mathrm{mg} \mathrm{g}^{-1} \mathrm{DW}\right)$}

Proline content was determined by the method of Bates et al., (1973).

\section{Procedure}

Leaf sample $(0.5 \mathrm{~g})$ was homogenized in $5 \mathrm{~mL}$ of sulphosalicylic acid (3\%). It was centrifuged at $6000 \mathrm{~g}$ for 10 minutes and supernatant was saved. Residue was again extracted twice with $5 \mathrm{~mL}$ of 3 per cent aqueous sulphosalicylic acid. All the supernatant fractions were pooled and final volume was made to $15 \mathrm{~mL} .2 \mathrm{~mL}$ of this extract was taken in the test tube and $2 \mathrm{~mL}$ ninhydrin reagent and $2 \mathrm{~mL}$ glacial acetic acid were added. The reaction mixture was put in boiling water bath for 30 minutes. After cooling the reaction mixture was added with $5 \mathrm{~mL}$ toluene. Then solution mixture was shaken vigorously and toluene fraction was separated by separating funnel. The absorbance of toluene fraction was read at $520 \mathrm{~nm}$ with the help of spectrophotometer against toluene blank. Concentration of proline in the plant samples was estimated by referring to a standard curve of proline.
Estimation of ascorbate peroxidase (APX) activity $\left(\mathrm{U} \mathrm{g} \mathrm{g}^{-1} \mathrm{FW} \mathrm{min}{ }^{-1}\right)$

\section{Enzyme extraction}

$100 \mathrm{mg}$ of leaves was homogenised in phosphate buffer in a pre chilled pestle and mortar. Homogenate was strained through two folds of muslin cloth and centrifuged at 16000 rpm for $20 \mathrm{~min}$ at $4^{0} \mathrm{C}$ and the supernatant was used as enzyme extract.

\section{Enzyme assay}

Ascorbate peroxidase activity was assayed according to the method as proposed by Nakano and Asada (1981). It was based on the decrease in absorbance at $290 \mathrm{~nm}$ as ascorbate was oxidized with time. The reaction mixture for the peroxidase contained $0.1 \mathrm{~mL}$ of $100 \mathrm{mM}$ potassium phosphate buffer $(\mathrm{pH} 7.5), 1.5 \mathrm{mM}$ $\mathrm{H}_{2} \mathrm{O}_{2}, 0.40 \mathrm{~mL}$ of $3 \mathrm{mM}$ ascorbic acid, $0.1 \mathrm{~mL}$ of $3 \mathrm{mM}$ EDTA and $1 \mathrm{~mL}$ of enzyme extract in a total volume of $3 \mathrm{~mL}$. In blank, ascorbic acid was not added. The reaction was started by adding the enzyme and $\mathrm{H}_{2} \mathrm{O}_{2}$ simultaneously and the decrease in absorbance was recorded 30 seconds after this addition at $290 \mathrm{~nm}$. The ascorbate peroxidase activity was estimated by referring to the standard curve.

\section{Estimation of protein}

Protein content of leaf samples was measured by using the method of Lowry et al., (1951).

\section{Results and Discussion}

Table 1 represents data regarding Superoxide dismutase (SOD) analysed at 20,40,60 and 80 DAT. Maximum SOD activity was noted at 40 DAT, after that reduction in SOD activity was noted. Primed sets showed higher SOD 
activity over non primed sets and maximum SOD activity was found in kinetin primed set $\mathrm{T}_{3}$. The increment in SOD activity was also noted under late sown condition. Same result was obtained in both consecutive years.

Data (Table 2.) regarding nitrate reductase activity(NR) of rice showed that NR activity was found to increase till 40 DAT, after that activity of NR decreases. Result showed that in late sown condition NR activity was found to decrease compared to timely sown condition. However primed sets showed higher NR activity over non primed control set and maximum NR activity was noted in set $\mathrm{T}_{2}$.. The trend was found similar for both the studied years.

Table 3 represents data regarding proline content in rice leaves, obtained from primed and non primed sets The proline content was found to increase with age of plant and maximum proline content was noted in $\mathrm{T}_{4}$ set. It was also found that primed sets showed higher proline content over non primed control set under both sowing conditions. Same result was observed in both the consecutive years. Table 4depicted the effect of seed priming on ascorbate peroxidase activity of leaves, under timely and late sown conditions at 20, 40, 60 and 80 DAT. Result showed that ascorbate peroxidase activity was found to increase with increasing plant age under both sowing conditions. Primed sets showed higher ascorbate peroxidase activity in comparison to non primed control set. The maximum ascorbate peroxidase activity was noted in $T_{4}$ set. It was also noted that activity of ascorbate peroxidise was found lesser in late sown condition. Similar result was obtained in both the consecutive years.

Table.1 Effect of hydro, halo and hormonal seed priming on superoxide dismutase activity (SOD) of rice leaf (Var. HUR 105)

\begin{tabular}{|c|c|c|c|c|c|c|}
\hline \multirow[t]{2}{*}{ Year } & \multirow{2}{*}{$\begin{array}{l}\text { Sowing } \\
\text { Time }\end{array}$} & \multirow[t]{2}{*}{ Treatments*** } & \multicolumn{4}{|c|}{ SOD activity (unit $\times 10^{2} \mathrm{~g}-{ }^{-} \mathrm{FW} \min ^{-1}$ ) } \\
\hline & & & 20 DAT & 40 DAT & 60 DAT & 80 DAT \\
\hline \multirow{10}{*}{2015} & \multirow{5}{*}{ I* } & $\mathrm{T}_{0}$ & $24.08 \pm 0.27^{j}$ & $40.45 \pm 0.50^{\mathrm{e}}$ & $53.18 \pm 0.59^{\mathrm{g}}$ & $77.25 \pm 0.81^{\mathrm{f}}$ \\
\hline & & $\mathrm{T}_{1}$ & $25.71 \pm 0.36^{\mathrm{h}, \mathrm{i}}$ & $42.48 \pm 0.58^{\mathrm{c}, \mathrm{d}}$ & $56.13 \pm 0.31^{\mathrm{f}}$ & $79.83 \pm 0.66^{\mathrm{e}}$ \\
\hline & & $\mathrm{T}_{2}$ & $27.77 \pm 0.33^{\mathrm{f}}$ & $45.18 \pm 0.63^{\mathrm{a}, \mathrm{b}}$ & $59.32 \pm 0.63^{\mathrm{e}}$ & $80.70 \pm 0.18^{\mathrm{d}, \mathrm{e}}$ \\
\hline & & $\mathrm{T}_{3}$ & $29.48 \pm 0.58^{a, b, c, d}$ & $45.55 \pm 0.52^{\mathrm{a}, \mathrm{b}}$ & $61.53 \pm 0.54^{\mathrm{a}, \mathrm{b}, \mathrm{c}}$ & $82.81 \pm 0.38^{\mathrm{b}, \mathrm{c}}$ \\
\hline & & T4 & $28.42 \pm 0.45^{\mathrm{e}, \mathrm{f}}$ & $44.91 \pm 0.38^{\mathrm{e}}$ & $60.77 \pm 0.40^{\mathrm{b}, \mathrm{c}, \mathrm{d}}$ & $80.76 \pm 0.31^{\mathrm{d}, \mathrm{e}}$ \\
\hline & \multirow{5}{*}{$\mathbf{I I}^{* *}$} & $\mathrm{~T}_{0}$ & $24.54 \pm 0.10^{j}$ & $40.95 \pm 0.41^{\mathrm{e}}$ & $53.62 \pm 0.52^{\mathrm{g}}$ & $77.58 \pm 0.67^{f}$ \\
\hline & & $\mathrm{T}_{1}$ & $26.42 \pm 0.29^{g, h}$ & $42.98 \pm 0.41^{\mathrm{c}}$ & $56.67 \pm 0.11^{f}$ & $80.69 \pm 0.12^{\mathrm{d}, \mathrm{e}}$ \\
\hline & & $\mathrm{T}_{2}$ & $28.32 \pm 0.26^{\mathrm{e}, \mathrm{f}}$ & $45.55 \pm 0.54^{\mathrm{a}, \mathrm{b}}$ & $59.78 \pm 0.62^{\mathrm{d}, \mathrm{e}}$ & $81.09 \pm 0.37^{\mathrm{d}, \mathrm{e}}$ \\
\hline & & $T_{3}$ & $29.85 \pm 0.32^{\mathrm{a}, \mathrm{b}, \mathrm{c}}$ & $45.85 \pm 0.37^{\mathrm{a}, \mathrm{b}}$ & $61.99 \pm 0.64^{\mathrm{a}, \mathrm{b}}$ & $83.35 \pm 0.28^{\mathrm{a}, \mathrm{b}}$ \\
\hline & & T4 & $29.05 \pm 0.35^{b, c, d, e}$ & $45.18 \pm 0.43^{\mathrm{a}, \mathrm{b}}$ & $61.19 \pm 0.28^{\mathrm{b}, \mathrm{c}}$ & $81.30 \pm 0.33^{\mathrm{d}}$ \\
\hline \multirow{10}{*}{2016} & \multirow{5}{*}{$\mathbf{I}^{*}$} & $\mathrm{~T}_{0}$ & $24.59 \pm 0.12^{j}$ & $40.92 \pm 0.28^{\mathrm{e}}$ & $53.65 \pm 0.55^{\mathrm{g}}$ & $77.82 \pm 0.61^{f}$ \\
\hline & & $\mathrm{T}_{1}$ & $26.36 \pm 0.30^{\mathrm{g}, \mathrm{h}}$ & $42.92 \pm 0.43^{c}$ & $56.45 \pm 0.36^{\mathrm{f}}$ & $80.51 \pm 0.15^{\mathrm{d}, \mathrm{e}}$ \\
\hline & & $\mathrm{T}_{2}$ & $28.47 \pm 0.35^{\mathrm{e}, \mathrm{f}}$ & $45.65 \pm 0.57^{\mathrm{a}, \mathrm{b}}$ & $59.69 \pm 0.61^{\mathrm{d}, \mathrm{e}}$ & $81.36 \pm 0.34^{\mathrm{d}}$ \\
\hline & & $\mathrm{T}_{3}$ & $29.95 \pm 0.36^{\mathrm{a}, \mathrm{b}}$ & $46.01 \pm 0.37^{\mathrm{a}, \mathrm{b}}$ & $61.98 \pm 0.40^{\mathrm{a}, \mathrm{b}}$ & $83.24 \pm 0.25^{\mathrm{a}, \mathrm{b}}$ \\
\hline & & $\mathrm{T} 4$ & $28.94 \pm 0.30^{\mathrm{c,d}, \mathrm{e}}$ & $45.37 \pm 0.31^{\mathrm{a}, \mathrm{b}}$ & $61.22 \pm 0.34^{\mathrm{b}, \mathrm{c}}$ & $81.33 \pm 0.39^{\mathrm{d}}$ \\
\hline & \multirow{5}{*}{ II $^{* * *}$} & $\mathrm{~T}_{0}$ & $24.93 \pm 0.34^{i, j}$ & $41.22 \pm 0.39^{\mathrm{d}, \mathrm{e}}$ & $54.05 \pm 0.36^{\mathrm{g}}$ & $77.83 \pm 0.38^{f}$ \\
\hline & & $\mathrm{T}_{1}$ & $26.71 \pm 0.04^{\mathrm{g}}$ & $43.26 \pm 0.24^{c}$ & $56.95 \pm 0.31^{\mathrm{f}}$ & $80.82 \pm 0.42^{\mathrm{d}, \mathrm{e}}$ \\
\hline & & $\mathrm{T}_{2}$ & $28.65 \pm 0.06^{\mathrm{d}, \mathrm{e}, \mathrm{f}}$ & $46.10 \pm 0.66^{\mathrm{a}, \mathrm{b}}$ & $60.37 \pm 0.28^{\mathrm{c}, \mathrm{d}, \mathrm{e}}$ & $81.72 \pm 0.03^{\mathrm{c}, \mathrm{d}}$ \\
\hline & & $\mathrm{T}_{3}$ & $30.19 \pm 0.28^{\mathrm{a}}$ & $46.42 \pm 0.33^{\mathrm{a}}$ & $62.68 \pm 0.14^{\mathrm{a}}$ & $84.26 \pm 0.71^{\mathrm{a}}$ \\
\hline & & $\mathrm{T} 4$ & $29.25 \pm 0.21^{\mathrm{a}, \mathrm{b}, \mathrm{c}, \mathrm{d}}$ & $45.37 \pm 0.30^{\mathrm{a}, \mathrm{b}}$ & $61.58 \pm 0.08^{\mathrm{a}, \mathrm{b}, \mathrm{c}}$ & $81.60 \pm 0.10^{c, d}$ \\
\hline
\end{tabular}

Data presented are means from three replicates with standard errors. Within each treatment, different letters indicate significant differences by Duncan's multiple range test at $\mathrm{P}<0.05$

*Timely sown

***ate sown

*** $\mathrm{T}_{0}$ : Non primed control; $\mathrm{T}_{1}$ : Hydro primed; $\mathrm{T}_{2}: \mathrm{Mg}\left(\mathrm{NO}_{3}\right)_{2} ; \mathrm{T}_{3}$ : Kinetin and $\mathrm{T}_{4}$ : Salicylic acid 
Table.2 Effect of hydro, halo and hormonal seed priming on nitrate reductase activity (NR) of rice leaf (Var. HUR 105)

\begin{tabular}{|c|c|c|c|c|c|c|}
\hline \multirow[t]{2}{*}{ Year } & \multirow{2}{*}{$\begin{array}{l}\text { Sowing } \\
\text { Time }\end{array}$} & \multirow[t]{2}{*}{ Treatments*** } & \multicolumn{4}{|c|}{ NR activity ( $\mathrm{n} \mathrm{mol} \mathrm{NO}_{2}^{-} \mathrm{h}^{-1} \mathrm{~g}^{-1} \mathrm{FW}$ ) } \\
\hline & & & 20 DAT & 40 DAT & 60 DAT & 80 DAT \\
\hline \multirow{10}{*}{2015} & \multirow{5}{*}{$I^{*}$} & $\mathrm{~T}_{0}$ & $360.83 \pm 3.05 \mathrm{i}, \mathrm{k}, \mathrm{i}$ & $1020.00 \pm 1.26^{\mathrm{h}}$ & $982.57 \pm 3.07^{f}$ & $743.60 \pm 0.67 \mathrm{~h}$ \\
\hline & & $\mathrm{T}_{1}$ & $374.33 \pm 1.99 \mathrm{~g}, \mathrm{~h}, \mathrm{i}$ & $1174.57 \pm 1.04 \mathrm{c}$ & $1082.40 \pm 2.34 \mathrm{c}$ & $817.50 \pm 1.57 c, d, e$ \\
\hline & & $\mathrm{T}_{2}$ & $417.37 \pm 2.00^{a}$ & $1288.57 \pm 4.25^{a}$ & $1175.70 \pm 2.66^{a}$ & $915.60 \pm 1.79 a$ \\
\hline & & $\mathrm{T}_{3}$ & $385.47 \pm 2.311^{e, f}$ & $1162.30 \pm 3.55^{d, e, f}$ & $1076.00 \pm 3.18 \mathrm{c}, \mathrm{d}, \mathrm{e}$ & $822.43 \pm 1.55^{c}$ \\
\hline & & T4 & $414.87 \pm 1.17 \mathrm{a}$ & $1172.13 \pm 2.32^{c, d}$ & $1078.83 \pm 1.72 c, d$ & $823.50 \pm 2.57 c$ \\
\hline & \multirow{5}{*}{ II $* *$} & $\mathrm{~T}_{0}$ & $356.33 \pm 2.78 \mathrm{k}, 1$ & $1009.47 \pm 2.32^{i, j}$ & $976.77 \pm 2.58^{f, g}$ & $738.70 \pm 1.78^{\mathrm{h}, \mathrm{i}}$ \\
\hline & & $\mathrm{T}_{1}$ & $369.53 \pm 2.07 \mathrm{~h}, \mathrm{~L}, \mathrm{j}$ & $1163.23 \pm 3.94^{\mathrm{d}, \mathrm{e}, \mathrm{f}}$ & $1078.43 \pm 1.65^{\mathrm{c}, \mathrm{d}}$ & $808.03 \pm 2.10^{f, g}$ \\
\hline & & $\mathrm{T}_{2}$ & $408.77 \pm 1.42^{\mathrm{a}, \mathrm{b}}$ & $1285.30 \pm 3.76^{a, b}$ & $1170.30 \pm 3.29 a, b$ & $909.50 \pm 2.31^{a}$ \\
\hline & & $\mathrm{T}_{3}$ & $381.50 \pm 2.17^{\mathrm{f}, \mathrm{h}}$ & $1157.87 \pm 3.95$ e,f,g & $1071.53 \pm 2.78^{\mathrm{d}, \mathrm{e}}$ & $816.30 \pm 2.93 \mathrm{c}, \mathrm{d}, \mathrm{e}, \mathrm{f}$ \\
\hline & & T4 & $403.77 \pm 1.15 \mathrm{~b}, \mathrm{c}$ & $1168.53 \pm 1.88^{c, d}$ & $1075.57 \pm 2.09 c, d, e$ & $820.73 \pm 2.89 \mathrm{c}, \mathrm{d}$ \\
\hline \multirow{10}{*}{2016} & \multirow{5}{*}{ I* } & $\mathrm{T}_{0}$ & $356.37 \pm 2.19 \mathrm{j}, \mathrm{k}$ & $1011.70 \pm 3.32 \mathrm{~d}$ & $979.77 \pm 3.00 \mathrm{~g}$ & $739.23 \pm 1.17 \mathrm{i}$ \\
\hline & & $\mathrm{T}_{1}$ & $370.70 \pm 1.19 \mathrm{~g}, \mathrm{~h}, \mathrm{i}$ & $1167.90 \pm 2.92 \mathrm{~b}, \mathrm{c}$ & $1078.77 \pm 2.30 \mathrm{c}, \mathrm{d}, \mathrm{e}$ & $809.83 \pm 2.95 \mathrm{f}, \mathrm{g}, \mathrm{h}$ \\
\hline & & $\mathrm{T}_{2}$ & $407.57 \pm 1.97 \mathrm{a}, \mathrm{b}$ & $1284.63 \pm 2.32^{b, c}$ & $1169.93 \pm 2.91^{\mathrm{a}, \mathrm{b}}$ & $906.53 \pm 2.62^{b}$ \\
\hline & & $\mathrm{T}_{3}$ & $378.47 \pm 3.99^{f, g}$ & $1158.97 \pm 5.14^{\mathrm{b}, \mathrm{c}}$ & $1072.13 \pm 3.41^{\mathrm{d}, \mathrm{e}, \mathrm{f}}$ & $816.50 \pm 1.05^{e, f}$ \\
\hline & & $\mathrm{T} 4$ & $406.80 \pm 3.00^{a, b, c}$ & $1167.83 \pm 2.28^{b}$ & $1075.13 \pm 0.86^{\mathrm{d}, \mathrm{e}, \mathrm{f}}$ & $823.10 \pm 2.96^{\mathrm{d}, \mathrm{e}}$ \\
\hline & \multirow{5}{*}{ II** } & $\mathrm{T}_{0}$ & $361.87 \pm 2.00 \mathrm{i}, \mathrm{j}$ & $1018.57 \pm 1.50^{d}$ & $981.17 \pm 3.77 \mathrm{~g}$ & $743.47 \pm 1.44^{i}$ \\
\hline & & $\mathrm{T}_{1}$ & $375.77 \pm 2.16 \mathrm{~g}, \mathrm{~h}$ & $1168.50 \pm 3.57 \mathrm{~b}, \mathrm{c}$ & $1083.57 \pm 1.55^{c}$ & $816.17 \pm 1.16^{\mathrm{e}, \mathrm{f}, \mathrm{g}}$ \\
\hline & & $\mathrm{T}_{2}$ & $416.40 \pm 0.85^{\mathrm{a}}$ & $1289.43 \pm 4.12^{\mathrm{a}}$ & $1174.87 \pm 3.32^{\mathrm{a}}$ & $917.80 \pm 1.92^{\mathrm{a}}$ \\
\hline & & $\mathrm{T}_{3}$ & $387.13 \pm 2.94$ e,f & $1165.10 \pm 5.18^{\mathrm{b}, \mathrm{c}}$ & $1076.53 \pm 2.34 \mathrm{c}, \mathrm{d}, \mathrm{e}, \mathrm{f}$ & $821.73 \pm 1.91^{\mathrm{d}, \mathrm{e}}$ \\
\hline & & T4 & $411.90 \pm 0.26^{a}$ & $1173.13 \pm 1.69 \mathrm{~b}, \mathrm{c}$ & $1080.23 \pm 1.85^{c, d}$ & $828.33 \pm 1.65^{\mathrm{d}}$ \\
\hline
\end{tabular}

Note: Detail of the conditions has given in table 1

Table.3 Effect of hydro, halo and hormonal seed priming on proline content of rice leaf (Var. HUR 105)

\begin{tabular}{|c|c|c|c|c|c|c|}
\hline \multirow[t]{2}{*}{ Year } & \multirow{2}{*}{$\begin{array}{l}\text { Sowing } \\
\text { Time }\end{array}$} & \multirow[t]{2}{*}{ Treatments*** } & \multicolumn{3}{|c|}{ Proline content $\left(\mu \mathrm{g} \mathrm{g}^{-1} \mathrm{DW}\right)$} & \multirow[b]{2}{*}{80 DAT } \\
\hline & & & 20 DAT & 40 DAT & 60 DAT & \\
\hline \multirow{10}{*}{2015} & \multirow{5}{*}{$\mathbf{I}^{*}$} & $\mathrm{~T}_{0}$ & $2.12 \pm 0.22 \mathrm{~d}, \mathrm{e}, \mathrm{f}$ & $9.98 \pm 0.30^{\mathrm{f}, \mathrm{g}}$ & $18.33 \pm 0.29 \mathrm{~g}, \mathrm{~h}$ & $20.18 \pm 0.17 \mathrm{~h}, \mathrm{i}$ \\
\hline & & $\mathrm{T}_{1}$ & $2.18 \pm 0.21^{d, e, f}$ & $10.45 \pm 0.00^{e, f}$ & $18.84 \pm 0.28^{\mathrm{f}, \mathrm{g}}$ & $20.90 \pm 0.35 \mathrm{~g}, \mathrm{~h}$ \\
\hline & & $\mathrm{T}_{2}$ & $2.81 \pm 0.03 \mathrm{a}, \mathrm{b}, \mathrm{c}, \mathrm{d}$ & $12.88 \pm 0.18^{\mathrm{b}, \mathrm{c}, \mathrm{d}}$ & $19.90 \pm 0.13^{c, d, e}$ & $23.96 \pm 0.32^{\mathrm{b}, \mathrm{c}}$ \\
\hline & & $\mathrm{T}_{3}$ & $2.74 \pm 0.06^{a, b, c, d}$ & $12.99 \pm 0.18^{\mathrm{b}, \mathrm{c}, \mathrm{d}}$ & $21.20 \pm 0.36^{b}$ & $23.08 \pm 0.34^{\mathrm{d}, \mathrm{e}, \mathrm{f}}$ \\
\hline & & $\mathrm{T} 4$ & $2.92 \pm 0.11^{\mathrm{a}, \mathrm{b}, \mathrm{c}}$ & $14.92 \pm 0.28^{a}$ & $22.96 \pm 0.25^{\mathrm{a}}$ & $25.82 \pm 0.37 \mathrm{a}$ \\
\hline & \multirow{5}{*}{$\mathbf{I I}^{* *}$} & $\mathrm{~T}_{0}$ & $1.69 \pm 0.34^{f}$ & $9.62 \pm 0.03 \mathrm{~g}$ & $17.71 \pm 0.43^{h}$ & $19.82 \pm 0.38^{\mathrm{i}}$ \\
\hline & & $\mathrm{T}_{1}$ & $1.91 \pm 0.20 \mathrm{e}, \mathrm{f}$ & $10.22 \pm 0.24 \mathrm{e}^{\mathrm{f}, \mathrm{fg}}$ & $18.31 \pm 0.13 \mathrm{~g}, \mathrm{~h}$ & $20.12 \pm 0.29 \mathrm{~h}, \mathrm{i}$ \\
\hline & & $\mathrm{T}_{2}$ & $2.43 \pm 0.03 \mathrm{~b}, \mathrm{c}, \mathrm{d}, \mathrm{e}$ & $12.37 \pm 0.13^{d}$ & $19.75 \pm 0.25 \mathrm{~d}, \mathrm{e}$ & $23.55 \pm 0.01^{b, c, d, e}$ \\
\hline & & $\mathrm{T}_{3}$ & $2.28 \pm 0.03 c, d, e, f$ & $12.45 \pm 0.06^{c, d}$ & $20.65 \pm 0.40^{\mathrm{b}, \mathrm{c}}$ & $22.41 \pm 0.07^{f}$ \\
\hline & & $\mathrm{T} 4$ & $2.91 \pm 0.42^{\mathrm{a}, \mathrm{b}, \mathrm{c}}$ & $14.42 \pm 0.07^{a}$ & $22.32 \pm 0.03^{a}$ & $25.42 \pm 0.03^{a}$ \\
\hline \multirow{10}{*}{2016} & \multirow{5}{*}{$I^{*}$} & $\mathrm{~T}_{0}$ & $2.24 \pm 0.11^{c, d, e, f}$ & $10.12 \pm 0.16^{\mathrm{f}, \mathrm{g}}$ & $18.28 \pm 0.03 \mathrm{~g}, \mathrm{~h}$ & $20.47 \pm 0.08 \mathrm{~g}, \mathrm{~h}, \mathrm{i}$ \\
\hline & & $\mathrm{T}_{1}$ & $2.73 \pm 0.09 \mathrm{a}, \mathrm{b}, \mathrm{c}, \mathrm{d}$ & $10.75 \pm 0.05^{\mathrm{e}}$ & $19.21 \pm 0.29 \mathrm{e}, \mathrm{f}$ & $21.09 \pm 0.28 \mathrm{~g}$ \\
\hline & & $\mathrm{T}_{2}$ & $2.78 \pm 0.24 \mathrm{a}, \mathrm{b}, \mathrm{c}, \mathrm{d}$ & $13.11 \pm 0.29 b, c$ & $20.02 \pm 0.29 c, d$ & $24.15 \pm 0.26^{\mathrm{b}}$ \\
\hline & & $\mathrm{T}_{3}$ & $2.88 \pm 0.29 \mathrm{a}, \mathrm{b}, \mathrm{c}$ & $13.22 \pm 0.33^{b}$ & $21.41 \pm 0.03^{b}$ & $23.14 \pm 0.32^{c, d, e, f}$ \\
\hline & & $\mathrm{T} 4$ & $3.36 \pm 0.24^{\mathrm{a}}$ & $15.02 \pm 0.34^{\mathrm{a}}$ & $23.12 \pm 0.35^{a}$ & $25.99 \pm 0.37 \mathrm{a}$ \\
\hline & \multirow{5}{*}{ II** } & $\mathrm{T}_{0}$ & $2.09 \pm 0.28 \mathrm{~d}, \mathrm{e}, \mathrm{f}$ & $9.79 \pm 0.26^{f, g}$ & $18.09 \pm 0.34 \mathrm{~g}, \mathrm{~h}$ & $20.04 \pm 0.40^{\mathrm{i}}$ \\
\hline & & $\mathrm{T}_{1}$ & $2.45 \pm 0.09 \mathrm{~b}, \mathrm{c}, \mathrm{d}, \mathrm{e}$ & $10.45 \pm 0.35^{e, f}$ & $18.72 \pm 0.07 \mathrm{f}, \mathrm{g}$ & $20.29 \pm 0.42 \mathrm{~g}, \mathrm{~h}, \mathrm{i}$ \\
\hline & & $\mathrm{T}_{2}$ & $2.73 \pm 0.08^{\mathrm{a}, \mathrm{b}, \mathrm{c}, \mathrm{d}}$ & $12.63 \pm 0.08 \mathrm{~b}, \mathrm{c}, \mathrm{d}$ & $19.95 \pm 0.36^{c, d, e}$ & $23.80 \pm 0.03 \mathrm{~b}, c, d$ \\
\hline & & $\mathrm{T}_{3}$ & $2.69 \pm 0.03 \mathrm{a}, \mathrm{b}, \mathrm{b}, \mathrm{d}$ & $12.98 \pm 0.18^{\mathrm{b}, \mathrm{c}, \mathrm{d}}$ & $20.85 \pm 0.27 \mathrm{~b}$ & $22.76 \pm 0.05^{\mathrm{e}, \mathrm{f}}$ \\
\hline & & $\mathrm{T} 4$ & $3.05 \pm 0.25^{a, b}$ & $14.72 \pm 0.09 \mathrm{a}$ & $22.72 \pm 0.06^{a}$ & $25.84 \pm 0.05^{a}$ \\
\hline
\end{tabular}

Note: Detail of the conditions has given in table 1. 
Table.4 Effect of hydro, halo and hormonal seed priming on ascorbate peroxidase activity of rice leaf (Var. HUR 105)

\begin{tabular}{|c|c|c|c|c|c|c|}
\hline \multirow[t]{2}{*}{ Year } & \multirow{2}{*}{$\begin{array}{l}\text { Sowing } \\
\text { Time }\end{array}$} & \multirow[t]{2}{*}{ Treatments*** } & \multicolumn{4}{|c|}{ Ascorbate peroxidase activity $\left(\mathrm{U} \mathrm{g}^{-1} \mathrm{FW} \mathrm{min}^{-1}\right)$} \\
\hline & & & 20 DAT & 40 DAT & 60 DAT & 80 DAT \\
\hline \multirow{10}{*}{2015} & \multirow{5}{*}{$\mathbf{I}^{*}$} & $\mathrm{~T}_{0}$ & $1.58 \pm 0.04^{\mathrm{i}}$ & $2.59 \pm 0.02^{\mathrm{i}}$ & $2.69 \pm 0.02^{\mathrm{k}, \mathrm{l}}$ & $2.84 \pm 0.02^{\mathrm{k}, \mathrm{l}}$ \\
\hline & & $\mathrm{T}_{1}$ & $1.70 \pm 0.03 \mathrm{~g}$ & $2.73 \pm 0.03 \mathrm{~g}$ & $2.83 \pm 0.02^{\mathrm{i}}$ & $2.98 \pm 0.02 \mathrm{j}$ \\
\hline & & $T_{2}$ & $2.54 \pm 0.03^{d}$ & $3.32 \pm 0.03 \mathrm{c}, \mathrm{d}, \mathrm{e}$ & $3.52 \pm 0.02^{\mathrm{d}, \mathrm{e}}$ & $3.86 \pm 0.01 \mathrm{~b}, \mathrm{c}, \mathrm{d}, \mathrm{e}$ \\
\hline & & $\mathrm{T}_{3}$ & $2.53 \pm 0.03^{d}$ & $3.28 \pm 0.05^{\mathrm{e}}$ & $3.50 \pm 0.02^{\mathrm{d}, \mathrm{e}}$ & $3.78 \pm 0.00^{\mathrm{e}, \mathrm{f}, \mathrm{g}}$ \\
\hline & & $\mathrm{T} 4$ & $2.69 \pm 0.04^{a, b}$ & $3.37 \pm 0.04 \mathrm{~b}, \mathrm{c}, \mathrm{d}$ & $3.56 \pm 0.01^{c, d}$ & $3.89 \pm 0.05^{b, c}$ \\
\hline & \multirow{5}{*}{ II** } & $\mathrm{T}_{0}$ & $1.46 \pm 0.02 j$ & $2.49 \pm 0.01 j$ & $2.65 \pm 0.02^{1}$ & $2.77 \pm 0.01^{1}$ \\
\hline & & $\mathrm{T}_{1}$ & $1.62 \pm 0.04^{\mathrm{h}, \mathrm{i}}$ & $2.64 \pm 0.02^{\mathrm{h}, \mathrm{i}}$ & $2.73 \pm 0.03 \mathrm{j}, \mathrm{k}$ & $2.81 \pm 0.03^{\mathrm{k}, \mathrm{l}}$ \\
\hline & & $\mathrm{T}_{2}$ & $2.43 \pm 0.02^{\mathrm{e}}$ & $3.17 \pm 0.04^{f}$ & $3.36 \pm 0.05^{f}$ & $3.67 \pm 0.04^{\mathrm{h}}$ \\
\hline & & $\mathrm{T}_{3}$ & $2.40 \pm 0.03 e$ & $3.11 \pm 0.02^{\mathrm{f}}$ & $3.28 \pm 0.02 \mathrm{~g}$ & $3.62 \pm 0.02^{\mathrm{h}}$ \\
\hline & & $\mathrm{T} 4$ & $2.60 \pm 0.03 \mathrm{c}, \mathrm{d}$ & $3.28 \pm 0.03 \mathrm{~d}, \mathrm{e}$ & $3.48 \pm 0.02^{\mathrm{e}}$ & $3.80 \pm 0.03 \mathrm{~d}, \mathrm{e}, \mathrm{f}$ \\
\hline \multirow{10}{*}{2016} & \multirow{5}{*}{ I* } & $\mathrm{T}_{0}$ & $1.68 \pm 0.02 \mathrm{~g}, \mathrm{~h}$ & $2.69 \pm 0.03 \mathrm{~g}, \mathrm{~h}$ & $2.76 \pm 0.01^{\mathrm{i} j, \mathrm{j}, \mathrm{k}}$ & $2.92 \pm 0.02 \mathrm{i}, \mathrm{k}$ \\
\hline & & $\mathrm{T}_{1}$ & $1.79 \pm 0.02^{\mathrm{f}}$ & $2.78 \pm 0.03 \mathrm{~g}$ & $2.93 \pm 0.01^{\mathrm{h}}$ & $3.19 \pm 0.03^{i}$ \\
\hline & & $\mathrm{T}_{2}$ & $2.66 \pm 0.02^{b, c}$ & $3.41 \pm 0.02^{a, b}$ & $3.64 \pm 0.02^{a, b}$ & $3.95 \pm 0.02^{a, b}$ \\
\hline & & $\mathrm{T}_{3}$ & $2.58 \pm 0.02^{c, d}$ & $3.37 \pm 0.02^{b, c}$ & $3.61 \pm 0.03^{b, c}$ & $3.82 \pm 0.01^{\mathrm{c}, \mathrm{d}, \mathrm{e}, \mathrm{f}}$ \\
\hline & & T4 & $2.77 \pm 0.02^{a}$ & $3.48 \pm 0.03 \mathrm{a}$ & $3.70 \pm 0.02^{\mathrm{a}}$ & $4.04 \pm 0.11^{a}$ \\
\hline & \multirow{5}{*}{ II** } & $\mathrm{T}_{0}$ & $1.59 \pm 0.02^{\mathrm{i}}$ & $2.59 \pm 0.02^{\mathrm{i}}$ & $2.75 \pm 0.02 \mathrm{i}, \mathrm{j}, \mathrm{k}$ & $2.84 \pm 0.01^{\mathrm{k}, 1}$ \\
\hline & & $\mathrm{T}_{1}$ & $1.71 \pm 0.03^{\mathrm{f}, \mathrm{g}}$ & $2.76 \pm 0.01 \mathrm{~g}$ & $2.80 \pm 0.03^{i, j}$ & $2.90 \pm 0.03 \mathrm{j}, \mathrm{k}$ \\
\hline & & $\mathrm{T}_{2}$ & $2.51 \pm 0.02 \mathrm{e}$ & $3.31 \pm 0.03 c, d, e$ & $3.49 \pm 0.05 \mathrm{~d}, \mathrm{e}$ & $3.76 \pm 0.03^{\mathrm{f}, \mathrm{g}, \mathrm{h}}$ \\
\hline & & $\mathrm{T}_{3}$ & $2.56 \pm 0.00^{d}$ & $3.27 \pm 0.03^{e}$ & $3.38 \pm 0.02^{f}$ & $3.72 \pm 0.04 \mathrm{~g}, \mathrm{~h}$ \\
\hline & & $\mathrm{T} 4$ & $2.68 \pm 0.04^{\mathrm{b}}$ & $3.41 \pm 0.04^{a, b}$ & $3.60 \pm 0.03 \mathrm{~b}, \mathrm{c}$ & $3.88 \pm 0.03 \mathrm{~b}, \mathrm{c}, \mathrm{d}$ \\
\hline
\end{tabular}

Note: Detail of the conditions has given in table 1 .

Table.5 Effect of hydro, halo and hormonal seed priming on protein content of rice leaf (Var.

HUR 105)

\begin{tabular}{|c|c|c|c|c|c|c|}
\hline \multirow[t]{2}{*}{ Year } & \multirow{2}{*}{$\begin{array}{l}\text { Sowing } \\
\text { Time }\end{array}$} & \multirow[t]{2}{*}{ Treatments*** } & \multicolumn{4}{|c|}{ Protein content $\left(\mathrm{mg} \mathrm{g}^{-1} \mathrm{FW}\right)$} \\
\hline & & & 20 DAT & 40 DAT & 60 DAT & 80 DAT \\
\hline \multirow{10}{*}{2015} & \multirow{5}{*}{$I^{*}$} & $\mathrm{~T}_{0}$ & $4.35 \pm 0.20^{\mathrm{h}, \mathrm{i}}$ & $9.85 \pm 0.26^{\text {def }}$ & $9.30 \pm 0.29 \mathrm{f}, \mathrm{g}, \mathrm{h}$ & $7.00 \pm 0.17 \mathrm{c}, \mathrm{d}$ \\
\hline & & $T_{1}$ & $4.75 \pm 0.20^{\mathrm{f}, \mathrm{g}}$ & $10.35 \pm 0.20 \mathrm{c}, \mathrm{d}, \mathrm{e}$ & $9.75 \pm 0.20^{\mathrm{d}, \mathrm{e}, \mathrm{f}, \mathrm{g}, \mathrm{h}}$ & $7.45 \pm 0.14^{b, c}$ \\
\hline & & $\mathrm{T}_{2}$ & $5.23 \pm 0.13 \mathrm{c}, \mathrm{d}, \mathrm{e}$ & $11.40 \pm 0.35^{\mathrm{a}, \mathrm{b}}$ & $10.79 \pm 0.51^{a, b, c, d}$ & $8.60 \pm 0.23^{a}$ \\
\hline & & $\mathrm{T}_{3}$ & $5.85 \pm 0.20^{\mathrm{a}}$ & $11.95 \pm 0.38^{a}$ & $11.53 \pm 0.36^{a}$ & $8.60 \pm 0.17 a$ \\
\hline & & $\mathrm{T} 4$ & $4.93 \pm 0.13^{\mathrm{e}, \mathrm{f}}$ & $10.25 \pm 0.20 \mathrm{~d}, \mathrm{e}, \mathrm{f}$ & $9.88 \pm 0.22 \mathrm{c}, \mathrm{d}, \mathrm{e}, \mathrm{f}, \mathrm{g}, \mathrm{h}$ & $7.15 \pm 0.09$ c.d \\
\hline & \multirow{5}{*}{ II $* *$} & $\mathrm{~T}_{0}$ & $4.38 \pm 0.07 \mathrm{~h}, \mathrm{i}$ & $9.80 \pm 0.12^{\mathrm{d}, \mathrm{e}, \mathrm{f}}$ & $9.25 \pm 0.09 \mathrm{~g}, \mathrm{~h}$ & $7.05 \pm 0.14 \mathrm{c}, \mathrm{d}$ \\
\hline & & $\mathrm{T}_{1}$ & $4.70 \pm 0.00^{\mathrm{f}, \mathrm{g}, \mathrm{h}}$ & $10.20 \pm 0.17 \mathrm{~d}, \mathrm{e}, \mathrm{f}$ & $9.63 \pm 0.04 \mathrm{e}, \mathrm{f}, \mathrm{g}, \mathrm{h}$ & $7.50 \pm 0.12^{b, c}$ \\
\hline & & $\mathrm{T}_{2}$ & $5.33 \pm 0.10^{b, c, d}$ & $11.70 \pm 0.12^{a, b}$ & $10.45 \pm 0.08 \mathrm{a}, \mathrm{b}, \mathrm{c}, \mathrm{d}, \mathrm{e}$ & $8.25 \pm 0.26^{a}$ \\
\hline & & $\mathrm{T}_{3}$ & $5.90 \pm 0.06^{a}$ & $11.85 \pm 0.09 \mathrm{a}$ & $11.20 \pm 0.06^{a}, \mathrm{~b}$ & $8.55 \pm 0.32^{a}$ \\
\hline & & $\mathrm{T} 4$ & $4.95 \pm 0.03 \mathrm{e}, \mathrm{f}$ & $10.50 \pm 0.23 c, d$ & $9.88 \pm 0.18 \mathrm{c}, \mathrm{d}, \mathrm{e}, \mathrm{f}, \mathrm{g}$ & $7.50 \pm 0.23 \mathrm{~b}, \mathrm{c}$ \\
\hline \multirow{10}{*}{2016} & \multirow{5}{*}{ I* } & $\mathrm{T}_{0}$ & $3.80 \pm 0.06^{\mathrm{k}}$ & $9.60 \pm 0.29 \mathrm{e}, \mathrm{f}$ & $9.08 \pm 0.33 \mathrm{~g}, \mathrm{~h}$ & $6.90 \pm 0.12^{c, d}$ \\
\hline & & $\mathrm{T}_{1}$ & $4.18 \pm 0.10^{i, j}$ & $9.95 \pm 0.32 \mathrm{~d}, \mathrm{e}, \mathrm{f}$ & $7.20 \pm 1.04^{i}$ & $7.25 \pm 0.09 \mathrm{c}, \mathrm{d}$ \\
\hline & & $\mathrm{T}_{2}$ & $4.93 \pm 0.13 \mathrm{e}, \mathrm{f}$ & $11.05 \pm 0.32^{\mathrm{b}, \mathrm{c}}$ & $10.48 \pm 0.51^{\mathrm{a}, \mathrm{b}, \mathrm{c}, \mathrm{d}, \mathrm{e}}$ & $8.30 \pm 0.29 a$ \\
\hline & & $\mathrm{T}_{3}$ & $5.50 \pm 0.12^{b, c}$ & $11.50 \pm 0.46^{a, b}$ & $11.25 \pm 0.38^{a, b}$ & $8.35 \pm 0.14^{a}$ \\
\hline & & $\mathrm{T}_{4}$ & $4.50 \pm 0.12 \mathrm{~g}, \mathrm{~h}, \mathrm{i}$ & $9.95 \pm 0.20 \mathrm{~d}, \mathrm{e}, \mathrm{f}$ & $9.75 \pm 0.03$ d,e,f,g,h & $7.15 \pm 0.09 \mathrm{c}, \mathrm{d}$ \\
\hline & \multirow{5}{*}{$\mathbf{I I}^{* *}$} & $\mathrm{~T}_{0}$ & $3.98 \pm 0.07 \mathrm{j}, \mathrm{k}$ & $9.50 \pm 0.00^{\mathrm{f}}$ & $9.00 \pm 0.12^{\mathrm{h}}$ & $6.65 \pm 0.03^{d}$ \\
\hline & & $\mathrm{T}_{1}$ & $4.50 \pm 0.06 \mathrm{~g}, \mathrm{~h}, \mathrm{i}$ & $9.95 \pm 0.20 \mathrm{~d}, \mathrm{e}, \mathrm{f}$ & $9.45 \pm 0.03^{\mathrm{fg}, \mathrm{h}}$ & $7.25 \pm 0.09 \mathrm{c}, \mathrm{d}$ \\
\hline & & $\mathrm{T}_{2}$ & $5.05 \pm 0.09 \mathrm{~d}, \mathrm{e}, \mathrm{f}$ & $11.50 \pm 0.12^{\mathrm{a}, \mathrm{b}}$ & $10.20 \pm 0.12^{b, c, d, e, f}$ & $8.00 \pm 0.23 \mathrm{a}, \mathrm{b}$ \\
\hline & & $\mathrm{T}_{3}$ & $5.62 \pm 0.05 \mathrm{a}, \mathrm{b}$ & $11.70 \pm 0.06^{\mathrm{a}, \mathrm{b}}$ & $10.90 \pm 0.00 \mathrm{a}, \mathrm{b}, \mathrm{c}$ & $8.55 \pm 0.20^{a}$ \\
\hline & & T4 & $4.80 \pm 0.00^{f, g}$ & $10.30 \pm 0.23 \mathrm{~d}, \mathrm{e}, \mathrm{f}$ & $9.75 \pm 0.15 \mathrm{~d}, \mathrm{e}, \mathrm{f}, \mathrm{g}, \mathrm{h}$ & $7.20 \pm 0.23 \mathrm{c}, \mathrm{d}$ \\
\hline
\end{tabular}

Note: Detail of the conditions has given in table 1. 
Table 5 represents protein content of rice leaves, obtained from primed and non primed sets at 20, 40, 60 and 80 DAT. Data showed that primed sets have more protein content over non primed control sets under both sowing conditions and maximum protein content was noted in $T_{3}$. It was also noted that protein content was found to increase in late sown condition. Same result was observed in both the consecutive years.

Antioxidant enzymes and metabolites increase under various environmental stress, with their comparatively higher activity in stress-tolerant cultivars, suggesting that higher antioxidant activity imparts tolerance (Polle 1997, Sairam and Saxena 2000). SOD is an enzyme that constitutes the first line of defence against ROS and it has already been established that seed priming with nitrate salts of $\mathrm{Mg}$ and $\mathrm{K}$ has shown an increment in SOD activity in wheat (Siddique et al., 2015). It may be due to the fact that, nitrate acts as a signalling molecule which triggers a number of changes in metabolic pathway (Bose and Pandey 2003, Magalhes et al., 2002). In the present study, late sowing of rice influence with various types of abiotic stresses cause accumulation of $\mathrm{H}_{2} \mathrm{O}_{2}$. It has been suggested that the accumulation of $\mathrm{H}_{2} \mathrm{O}_{2}$ levels caused by various environmental stresses would result in the combined activity of catalase and ascorbate peroxidase in order to protect plant cells. (Sofo et al., 2015).Bose et al.(2016) reported that rice seed priming with nitrate salts significantly improved SOD and NR activity in leaves under deferent sowing conditions. Pre sowing and soaking treatment in Brassica juncea seed with magnesium salts significantly induce growth, nitrate reductase activity, total protein content and yield (Bose and Mishra, 1999).

From the above study, we concluded the significant role of seed priming in rice seed which could mitigate the deleterious affect of abiotic stress by increasing the antioxidant activity and biochemical contents under late sown and timely sown conditions.

\section{References}

Abou-Khalifa AA (1996). Effect of some cultural treatment on rice. Ms. Thesis, Fac. Agric. Moshtohor, Zagazig Univ

Abou-Khalifa, A.A., 2005. Physiological behavior of same rice cultivars under different sowing dates and seedling ages. The 11th con. Argon. Dept., Fac., Agric., Assiut Univ., Nov, pp.1516.

Anaytullah, Bose B (2007) Nitrate-hardened seeds increase germination, amylase activity and proline content in wheat seedlings at low temperature. Physiol Mol Biol Plants 13:199207

Bates, L.S., Waldran, R.P. and Teare, I.D., Rapid determination of free proline for water stress studies, Plant Soil, 39, 205-208, 1973.

Bose B, Kumar R, Kuril S K and Srivastava A K (2007). Hardening of mustard seeds with $\mathrm{Mg}(\mathrm{NO} 3) 2$ increases seed germination, vegetative growth, nitrogen assimilation and yield. Brassica 9: 33-38.

Bose, B. and Mishra, T., Influence of presowing soaking treatment insome Brassica Juncea seeds with Mg-salts on growth, nitrate reductase activity, total protein content and yield responses, Physiol. Mol. Biol. Plants, 5,83-88, 1999.

Bose, B. and Panday, M.K., Effect of nitrate pre soaking of okra (Abelmoschus esculentus L.) seeds on growth and nitrate assimilation of seedling, Physiol. Mol. Biol. Plants., 5, 83-89, 2003.

Bose, B., Srivastava, A.K. and Siddique, A., 2016. Impact of nitrate salt hardened seeds and sowing dates on seedling stand, growth, yield attributes, nitrogen and stress metabolism of rice. International Journal of Agriculture, Environment and Biotechnology, 9(3), p.381.

Chen, Keting, Arora R (2011) Dynamics of the antioxidant system during seed osmopriming, post-priming germination, and seedling establishment in Spinach (Spinacia oleracea). Plant Science. 180: 212-220.

Dhindsa, R.A., Plumb-Dhindsa, P. and Thorpe, T.A., Leaf senescence: correlated with increased permeability and lipid peroxidation, and 
decreased level of super oxide dismutase and catalase, J. Exp. Bot., 126, 93-10, 1981.

Farooq M, Basra SMA, Rehman H, Saleem BA (2008) Seed priming enhances the performance of late sown wheat (Triticumaestivum L.) by improving chilling tolerance. J. of Agronomy and Crop Science. 194: 55-60.

Iqbal, M. and Ashraf, M., 2007. Seed preconditioning modulates growth, ionic relations, and photosynthetic capacity in adult plants of hexaploid wheat under salt stress. Journal of plant nutrition, 30(3), pp.381-396.

Korkmaz, Ahmet, Korkmaz Y (2009) Promotion by 5-aminolevulenic acid of pepper seed germination and seedling emergence under low-temperature stress. Scientia horticulturae. 119: 98-102.

Krishnotar, Bose B, Srivastava AK, Shahi JP (2009) Response of rabi maize crop to seed invigoration with magnes ium nitrate and distilled water. Indian J Plant Physiol. 14:7177.

Kumar M, Pant B, Mondal S, Bose B (2016) Hydro and halo priming: influenced germination responses in wheat Var-HUW-468 under heavy metal stress. Acta physiologiae plantarum, 38;217.

Lowry, O.H., Rosebrough, N.J., Farr, A.L. and Randall, R.J., 1951. Protein measurement with the Folin phenol reagent. Journal of biological chemistry, 193(1), pp.265-275.

Magalhaes, J.R., Monte, D.C. and Durzan, D., Nitric oxide and ethylene emission in Arabidopsis thaliana, Physiology and Molecular Biology of Plants, 6, 117-127, 2000

Nakano, Y. and Asada, K., 1981. Hydrogen peroxide is scavenged by ascorbate-specific peroxidase in spinach chloroplasts. Plant and cell physiology, 22(5), pp.867-880.

Polle, A., 1997. Defense against photooxidative damage in plants. Cold Spring Harbor Monograph Series, 34, pp.623-666.

Sairam, R.K., Srivastava, G.C. and Saxena, D.C., 2000. Increased antioxidant activity under elevated temperatures: a mechanism of heat stress tolerance in wheat genotypes. Biologia Plantarum, 43(2), pp.245-251.

Sananda M, Bose B (2012) Kinetics studies on $\alpha$ amylase extracted from germinating wheat endosperm of primed and non-primed seeds. Indian Journal of Agricultural Biochemistry, 25:137-141

Sharief AE, El-Hinidi MH, Abd El-Rahman AA, Abdo GM (2000). Rice productivity as influenced by planting dates and seedling. Age J. Ages. J. Agric. Sci. Mansoura Univ. 3:15111521

Siddique, A. and Bose, B., 2015. Effect of Seed Invigoration with Nitrate Salts on MorphoPhysiological and Growth Parameters of Wheat Crop Sown in Different Dates in its Cropping Season. Vegetos-An International Journal of Plant Research, 28(1), pp.76-85.

Sofo, A., Scopa, A., Nuzzaci, M. and Vitti, A., 2015. Ascorbate peroxidase and catalase activities and their genetic regulation in plants subjected to drought and salinity stresses. International journal of molecular sciences, 16(6), pp.13561-13578.

Srivastava AK, Bose B (2012) Effect of nitrate seed priming on Phenology, crop growth rate and yield attributes in rice (Oryza sativa L.). Vegetos. 25:174-181.

Srivastava, H.S., 1974. In vivo activity of nitrate reductase in maize seedlings. Indian journal of biochemistry \& biophysics, 11(3), pp.230-232.

Zhou ZS, Guo K, Elbaz AA, Yang ZM (2009) Salicylic acid alleviate mercury toxicity by preventing oxidative stress in roots of Medicago sativa. Environ Exp Bot 65:27-34.

\section{How to cite this article:}

Mahesh Kumar, Ravi P. Singh and Bandana Bose. 2018. Effect of Seed Priming on the Activity of Antioxidant Enzymes and Changes of Biochemicals of Rice under Timely and Late Sown Conditions. Int.J.Curr.Microbiol.App.Sci. 7(05): 2985-2992.

doi: https://doi.org/10.20546/ijcmas.2018.705.347 